\section{Factor VII deficiency in a patient with retinal arteriolar tortuosity syndrome}

\begin{abstract}
Purpose To report a pedigree with hereditary retinal arteriolar tortuosity with macular haemorrhage and abnormality of the coagulation system.

Methods Case report and literature review. Results A 49-year-old woman was referred due to macular haemorrhage in both eyes. Her 16-year-old son had recurrent retinal haemorrhages which presented at age 16 years and had mild retinal arteriolar tortuosity. Coagulation studies in the son revealed normal activated partial thromboplastin time (APTT), prolonged prothrombin time (PT) and $30 \%$ activity of factor VII.

Conclusions Factor VII deficiency may aggravate the haemorrhages in retinal arteriolar tortuosity syndrome. We therefore suggest conducting routine coagulation studies (PT, APTT) in all patients with retinal arteriolar tortuosity syndrome. Determination of factor VII activity is warranted only in patients with normal APTT and prolonged PT.
\end{abstract}

Key words Arteriolar tortuosity, Factor VII, Haemorrhage

Hereditary retinal arteriolar tortuosity (RAT) is a rare, well-recognised syndrome characterised by tortuosity of the retinal arterioles which increases with age. ${ }^{1}$ Patients usually present with acute loss of vision from a macular haemorrhage that resolves without sequelae. The pathogenesis of the retinal arteriolar tortuosity and the spontaneous haemorrhages in the macula is not clear, and no abnormalities of the coagulation system supporting a haemorrhagic diathesis have been reported to date.

We describe two patients, a mother and son, with RAT and macular haemorrhage, where the son also had a factor VII deficiency. The relationship between the two entities is discussed.
DAVID ZADOK, YAIR LEVI, HAYA ZEHAVI, NAOMI RAHIMI-LEVENE, PINHAS NEMET, ABRAHAM KORNBERG

Case reports

Case 1

An otherwise healthy 16-year-old boy presented in 1994 with a 2 day history of sudden visual loss in the right eye. He denied any history of trauma or bleeding tendency. Past ocular history and medical examination were unremarkable. His parents were unrelated. His best corrected visual acuity was $20 / 60$ in the right eye and 20/20 in the left. There was no relative afferent pupillary defect. Intraocular pressure and slit-lamp examination of the anterior segment were normal bilaterally. Fundus examination of both eyes showed mild RAT (Fig. 1a,b). The venous tree was normal. The right eye had a macular haemorrhage of the subretinal limiting membrane that explained the visual loss. Prothrombin time (PT) was $18.1 \mathrm{~s}$ (normal 12.8-15.0 s), and the international normalised ratio (INR) was 1.85 (normal 0.9-1.15). Activated partial thromboplastin time (APTT) was normal. Factor VII activity was 30\% (normal 60-120\%). Other coagulation studies, including platelet count, thrombin time, bleeding time, fibrinogen and activity of factors II, V, IX and X, were normal. Haemoglobin, haematocrit, leucocyte count, liver enzymes, serum proteins and creatinine were also normal. The retinal haemorrhages resolved without treatment. During the next 4 years he had three more episodes of macular haemorrhages that also resolved spontaneously. Retinal arteriolar tortuosity increased during this 4 year period (Fig. 1c).

\section{Case 2}

The 49-year-old mother of case 1 presented with sudden visual loss in her left eye. Her past ocular history was unremarkable. She had arterial hypertension. Her best corrected visual acuity was 20/30 in the right eye and 20/200 in the left. There was no relative afferent pupillary defect. Intraocular pressure and slit-lamp examination of the anterior segment were normal bilaterally. Fundus examination of both eyes was remarkable for RAT. The left eye had macular haemorrhages (Fig. 2). Coagulation studies were normal, including platelet count,
D. Zadok

Y. Levi

H. Zehavi

P. Nemet

Department of

Ophthalmology

Assaf Harofeh Medical

Center

Zerifin, Israel

N. Rahimi-Levene

A. Kornberg

Department of Hematology

Assaf Harofeh Medical

Center

Zerifin, Israel

David Zadok, MD

Department of

Ophthalmology

Assaf Harofeh Medical

Center

Zerifin 70300 , Israel

Tel: +97289779355

Fax: +972 89779361

Received: 11 October 1999 Accepted in revised form: 21 March 2000 


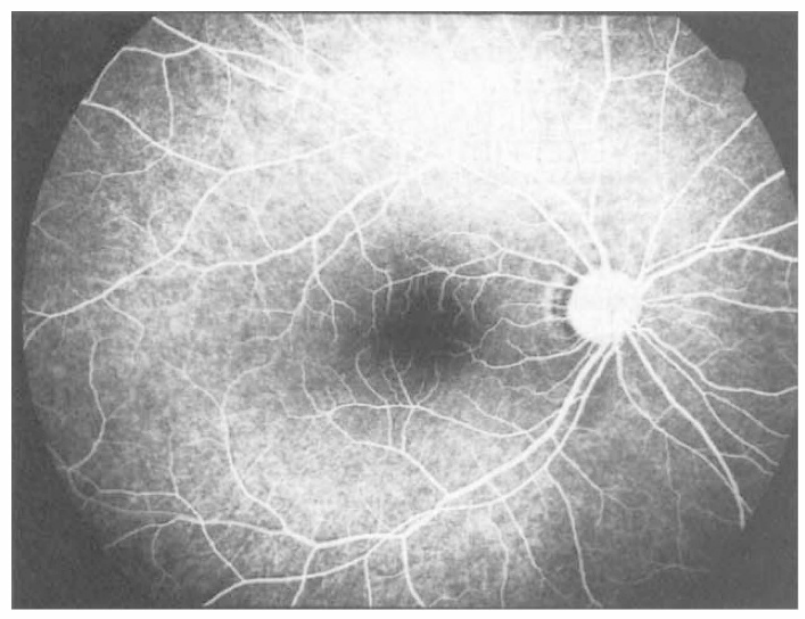

(a)

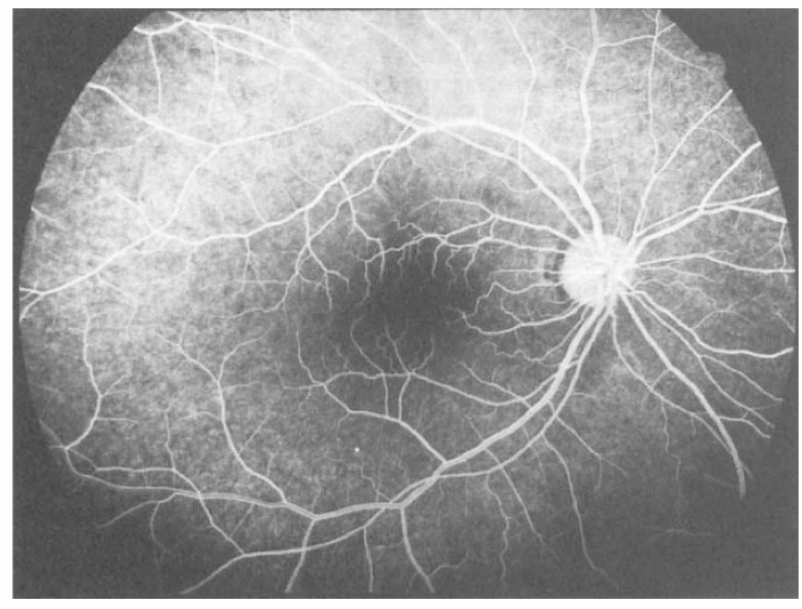

(c)

PT, APTT, thrombin time, bleeding time, and activity of factors II, V, VII, IX and X. The macular haemorrhages resolved spontaneously. She experienced no additional haemorrhages during the next 2 years.

\section{Discussion}

Familial retinal arteriolar tortuosity with retinal haemorrhages was first described by Beyer in $1958 .^{2}$ This syndrome is autosomal dominantly inherited, but the exact chromosomal localisation is unknown. RAT is characterised by tortuosity of the retinal arterioles, which is often subtle and easily overlooked and may increase with age to an easily discernible magnitude. ${ }^{1}$ The tortuosity is more apparent in medium and small arterioles, and more prominent in macular than extramacular arterioles. ${ }^{1}$ The retinal veins remain normal. Patients generally present with acute loss of vision from a macular haemorrhage that resolves without sequelae. The haemorrhages are usually small, superficial, circular or flame-shaped, and may be intraretinal or preretinal. ${ }^{3}$ Retinal haemorrhages in RAT are usually spontaneous, but may be related to physical exertion, sudden elevated blood pressure or mild trauma. ${ }^{4,5}$

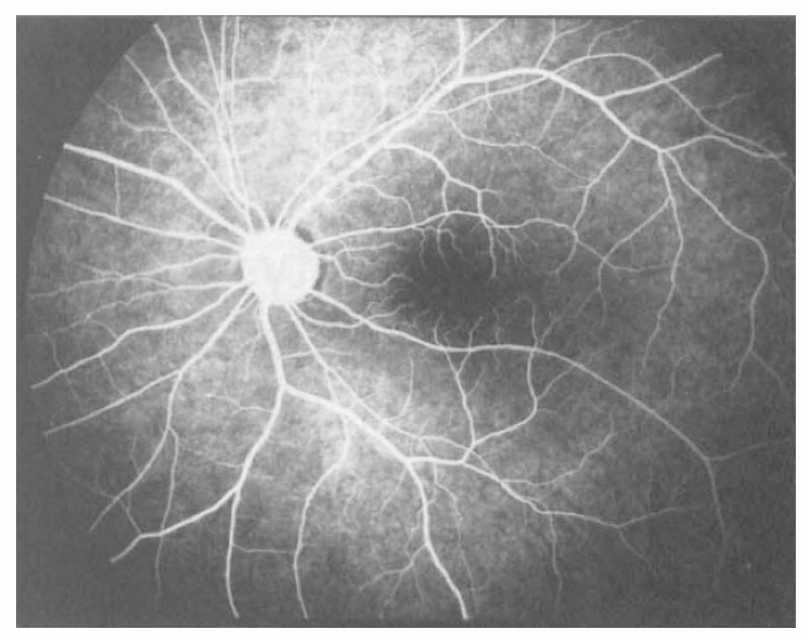

(b)

Fig. 1. Case 1. Fluorescein angiograms. At age 16 years mild retinal arteriolar tortuosity is present in both the right (a) and left (b) eye. No changes are apparent in the veins. At age 20 years there is increased retinal arteriolar tortuosity compared with age 16 years in the right eye (c).

The pathogenesis of macular haemorrhage in patients with RAT is unclear. ${ }^{1}$ A structural weakness in the perimacular vasculature was suggested, but the only abnormal histological finding of questionable significance in one patient was a focal increase in the number of endothelial cells in the retinal capillaries.,

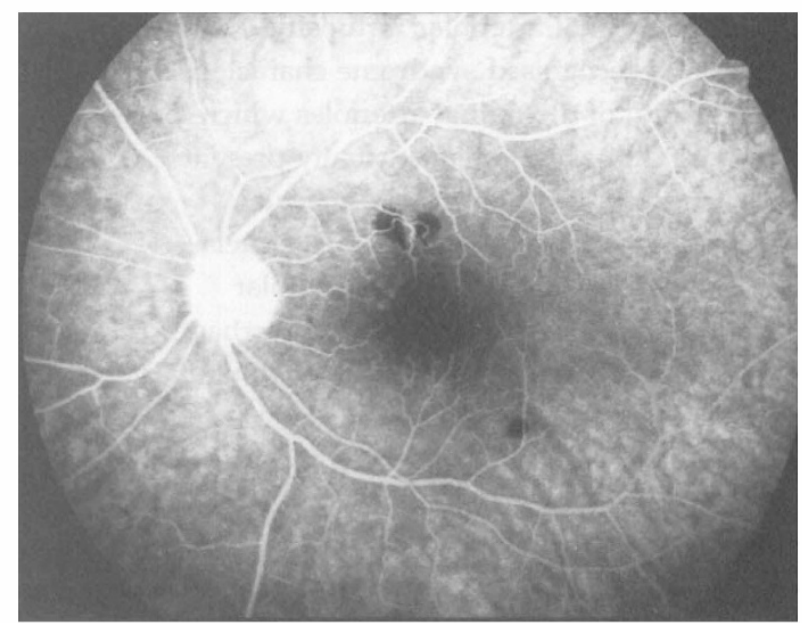

Fig. 2. Case 2. Fluorescein angiogram of the left eye at age 49 years shows macular haemorrhages and retinal arteriorlar tortuosity. 
No consistent abnormalities of the coagulation system have been reported in RAT syndrome. Platelet disorder was suggested but not proved in one patient. ${ }^{6}$

The sole clinical and laboratory abnormality in our 16-year-old patient was a prolonged PT due to factor VII deficiency. This deficiency should be considered when PT is prolonged and APTT is normal. ${ }^{7}$ We therefore suggest conducting routine coagulation studies (PT, APTT) in all patients with retinal arteriolar tortuosity syndrome. Determination of factor VII activity is warranted only in patients with normal APTT and prolonged PT.

Factor VII deficiency may be hereditary or acquired. ${ }^{8}$ Factor VII activity of the mother was normal; the boy's father and brothers were unavailable for evaluation. The factor deficiency was considered hereditary in the boy in view of the absence of liver or biliary system disease and malabsorption, a standard diet, normal activity of other vitamin-K-dependent factors, and no drug ingestion.

Factor VII deficiency is inherited as an autosomal recessive trait. It affects males and females equally with an incidence of 1 in $500000 .^{9}$ The gene for factor VII is located on chromosome 13, and most cases of factor VII deficiency are due to point mutations or partial gene deletions. The minimal haemostatic level of the factors is not clear. Generally, $15-25 \%$ of normal results in normal haemostasis. ${ }^{8}$ However, there are reports of patients with factor VII activity of $20-30 \%$, as in our patient, who developed intracranial, ${ }^{10}$ subararchnoid $^{7}$ and postpartum haemorrhages. ${ }^{8}$

The different clinical courses of the RAT syndrome in the mother and son, in which the boy suffered more episodes of intraocular haemorrhages, together with the factor VII deficiency only in the boy, suggest a causal relationship between the factor deficiency and the propensity to the haemorrhages. The factor deficiency might have aggravated the haemorrhagic diathesis in the boy.

The retinal haemorrhages in the boy resolved spontaneously without treatment to correct the coagulation abnormality. Individuals with more severe factor VII deficiency may require replacement with plasma or prothrombin complex to overcome the bleeding. Thus, a complete coagulation study is warranted in patients with RAT syndrome.

The genetic defect leading to RAT syndrome, and the incidence of RAT and factor VII deficiency in the same patient, are unknown. While their coexistence may be purely coincidental, it is possible that the genetic defect leading to RAT syndrome is related to that responsible for factor VII deficiency. This hypothesis requires further study.

\section{References}

1. Wells CG, Kalina RE. Progressive inherited retinal arteriolar tortuosity with spontaneous retinal hemorrhages. Ophthalmology 1985;92:1015-24.

2. Beyer EM. Familiaire Tortuositas der kleinen Netzhautarterien mit Makulablutung. Klin Monatsbl Augenheilkd 1958;132:532-9.

3. Kalina RE, Kaiser M. Familial retinal hemorrhages. Am J Ophthalmol 1972;74:252-5.

4. Goldberg MF, Pollack IP, Green WR. Familial retinal arteriolar tortuosity with retinal haemorrhage. Am J Ophthalmol 1972;73:183-91.

5. Faisal ZK, Abboud EB. New hemorrhages during scleral buckling in inherited retinal arteriolar tortuosity. Arch Ophthalmol 1995;113:853-4.

6. Werner H, Gafner F. Beitrag zur familiaren Tortuositas der kleinen Netzhautarterien. Ophthalmologica 1961;141:350-3.

7. Papa ML, Schisano G, Franco A, Nina P. Congenital deficiency of factor VII in subarachnoid hemorrhage. Stroke 1994;25:508-10.

8. Roberts HR, Hoppman M. Hemophilia and related conditions: inherited deficiencies of prothrombin, factor $\mathrm{V}$ and factor VII to XII. In: Betler E et al., editors. Williams hematology. 5th ed. New York: McGraw-Hill, 1995:1431-2.

9. Furie B, Furie B. Molecular and cellular biology of blood coagulation. N Engl J Med 1992;326:800-6.

10. Seaborg BJ, Burns CP, Olson JD, Pennell BJ, Follett KA. Intracranial hemorrhage in an adult with factor VII deficiency. Am J Hematol 1994;45:98-9. 